\title{
Knowledge, Attitude and Practice among Postnatal Mothers Regarding Postnatal Exercise in Selected Villages at Pooncheri, Kanchipuram District,Tamil Nadu, India
}

\author{
K. Mageshwari ${ }^{1}$, D. Pavithra ${ }^{1}$, C. Suniliga ${ }^{1}$, M. Priyanka ${ }^{1}$, D. Joaniepriya ${ }^{2}$ \\ ${ }^{1}$ B.Sc. (Nursing) IV Year Students, ${ }^{2}$ Guide, M.Sc. (Nursing) Assistant Professor, Department of Obstetrics and \\ Gynecological Nursing, Chettinad College of Nursing, Chettinad Academy of Research and Education, Rajiv \\ Gandhi Salai, Kelambakkam, Kanchipuram District, Tamil Nadu
}

\begin{abstract}
A descriptive study to assess the knowledge, attitude and practice among postnatal mothers regarding postnatal exercise in selected village adopted by rural health centrepooncheri, Kanchipuram District, Tamil Nadu, India. The objectives are to assess the knowledge, practice and attitude among postnatal mothers regarding postnatal exercise in selected villages adopted by rural health centrepooncheri, Kanchipuram District, Tamil Nadu, India . To find out the association between level of knowledge, practice and attitude with demographic variables. The purposive sampling technique was used and the samples were 73 . The data collection tools were validated and reability was established. The data were collected by self-administered questionnaire. The collected data tabulated and analyzed. Descriptive and inferential statistics were used. The study shows, In knowledge, $12.3 \%$ of the mothers had adequate knowledge, $52 \%$ of mothers had moderate knowledge and $35.6 \%$ of mothers had inadequate knowledge. In practice, $1.3 \%$ of mothers had adequate practice, $38.3 \%$ of mothers had moderate practice and $60.2 \%$ of mothers had inadequate practice, In attitude, $60.2 \%$ of mothers had negative attitude, $39.7 \%$ of mothers had undecided attitude and none of mothers had positive attitude among postnatal exercise.
\end{abstract}

Keywords: Postnatal exercise, postnatal mothers.

\section{Introduction}

Child birth is a significant event in women. It is a privilege the mother to deliver a baby woman role is completing after child birth. Childbirth is the culmination of a human pregnancy or gestation period with birth of one or more newborn infants from a women's uterus. The process of normal human childbirth is categorized

\section{Corresponding Author:}

\section{Pavithra,}

B.Sc. (Nursing) IV Year student Department of

Obstetrics and Gynecological Nursing, Chettinad

College of Nursing, Chettinad Academy of Research

and Education, Kelambakkam, Kanchipuramdist, Tamil

Nadu-603103

e-mail: pavithradeventhiran18@gmail.com

Phone No.: 8610918278 in the three stages of labour. The shorting and dilation of the cervix, descent and birth of the infant and birth of the placenta. In some cases, childbirth is achieved through caesarean section, the removal of the neonate through a surgical incision in the abdomen, rather than through vaginal birth. ${ }^{[1]}$ (Rajiya.H, 2011)

WHO 2008 reports, the outcome on the full range of issues relevant to the postpartum period for the mother and newborn which is a comprehensive view of maternal and newborn needs at a time which is decisive for the life and health of both the mother and her newborn. Taking women own perception of their needs during this period, it is major maternal and neonatal health challenges.

A number mothers experience to trouble bringing their abdomen into its original tone and size after childbirth. Some exercise can help our abdomen return to its original size. There has been gradual acceptance that exercise is beneficial during any part of reproductive 
process. Benefits are particularly apparent for those women exercising postpartum, where exercise have been found to improve weight loss and prevent long term weight retention.

Specific postnatal exercise is fairly important and involves re-strengthening and toning weakened abdomen, lower and upper back muscles. A series of physical exercise that are performed by the mother is to bring about optimal functioning of all system and prevent complication. ${ }^{[2]}$ (Shelin Christie, 2014)

Postnatal exercise offers a wide range of benefits for the mother. Postnatal exercise will speed up the recovery process and build valuable strength specific exercise for abdominal wall is transverse abdominal muscle which is used in the treatment of back pain. The specific exercise approach aims to improve the dynamic stability role of the local muscle in providing stiffness to the segments of the spine and pelvic during functional posture and movements. ${ }^{[3]}$ (Asha Sreenivasan, 2017)

The postnatal period is the time during which mothers body adjusts physically and psychologically to the process of childbearing. The period following childbirth during which the body tissues, in particular the genital and the pelvic organs, return to the condition they were pre-pregnancy, which lasts 6 weeks. Physiological changes during this time include: The cardiovascular system reverts, the vaginal wall is initially swollen, bluish and pouting but rapidly regains its tone although remaining fragile for 1-2 weeks, perineal edema may persist for some days. Biologically, Postnatal period is a time during which the mothers body including hormone levels and uterus size returns to pre-pregnancy conditions. ${ }^{[1]}$ (Rajiya.H,2011)

\section{Research Methodology}

The chapter deals with a brief description of different steps undertaken by the researchers for the study. It involves research approach, design the setting, the population, sample and criteria, sampling technique, sample size, data collection tool, score and interpretation and ethical consideration.

Research Approach: It involves the description of the plan to investigation the phenomenon under study in a structured, unstructured, or a combination of the two method (quantitative-qualitative integrated approach).
In this study, researchers assessed the knowledge, practice and attitude of postnatal exercise among postnatal mothers by using Quantitative approach.

Research Design: Research design can be defined as a blue print to conduct a research study, which involves the description of research approach, study setting, sampling size, sampling technique, tools and method of data collection and analysis to answer specific research questions or for testing research hypothesis.

Descriptive research design was used for the present study.

Research Setting: The physical location and condition in which data collection takes place in a study.

The study was conducted in selected villages adopted by rural health centre Pooncheri, Kanchipuram District, Tamil Nadu, India.

Population: The entire set of individuals or objects having some common characteristic(s) selected for a research study.

The present study comprises of all the postnatal mothers.

Target Population: The entire population in which the researchers are interested and to which they would like to generalize the research findings.

All the postnatal mothers in a selected villages adopted by rural health centre .

Accessible Population: The aggregate of cases that conform to designated are inclusion or exclusion criteria and that are accessible as subjects of the study.

The population of the present study comprises of all postnatal mothers who were all available during the period of the data collection in the selected villages adopted by rural health centre.

Sample: All the postnatal mothers in a selected villages.

Sample Criteria: List of the characteristics essential for inclusion or exclusion in the target population.

Inclusion Criteria: Postnatal mothers available at the time of data collection

Postnatal mothers who can understand Tamil 
Exclusion Criteria: Postnatal mothers who are not willing to participate.

Sample Technique: Sampling is the process of selecting a representative part of the population. Thus a carefully carried out sampling process helps to draw a sample that represents the characteristics of the population from which the sample is drawn. There are several method or techniques of sampling; however, basically sampling techniques are classified in two broad categories:

1. Probability sampling technique

2. Non-probability sampling technique

The purposive sampling technique was adopted for this study.

Sample Size: The sample size $=73$

Data Collection Tool: Identification of subjects and the precise, systematic gathering of information (data) relevant to the research purpose or the specific objectives, questions, or hypothesis of a study.

A self-structured questionnaire was used as a tool for data collection, it was consisted the following parts.

Part-1: Questions related to demographic profile of postnatal mothers. Age, Educational status, Occupation, family income, type of family, number of pregnancy, type of delivery, number of children, source of information.

Part-2: Self-structured questionnaire to assess the knowledge practice and attitude it consist of 30 questions each sample.

Score and Interpretation: Each correct answer was given 1 mark.

Table 1: Total number of knowledge questions-10, Total number of practice questions-10

\begin{tabular}{|l|l|}
\hline Level of Knowledge and Practice & Score \\
\hline Adequate knowledge and practice & $75 \%$ and above \\
\hline Moderate knowledge and practice & $51-75 \%$ \\
\hline Inadequate knowledge and practice & Below $50 \%$ \\
\hline
\end{tabular}

Table 2: Total number of attitude questions-10

\begin{tabular}{|l|l|}
\hline Level of Attitude & Score \\
\hline Positive attitude & $75 \%$ and above \\
\hline Undecided attitude & $51-75 \%$ \\
\hline Negative attitude & Below $50 \%$ \\
\hline
\end{tabular}

Table 3: Five point scale scoring interpretation

\begin{tabular}{|l|c|}
\hline Attitude & Score \\
\hline Strongly agree & 5 \\
\hline Agree & 4 \\
\hline Undecided & 3 \\
\hline Disagree & 2 \\
\hline Strongly disagree & 1 \\
\hline
\end{tabular}

Statistical Analysis: Descriptive and Inferential statistical analysis was used to analyze the data.

Ethical Consideration: UG Committee clearance was obtained.

Human Ethical committee clearance was obtained.

Prior permission was obtained from village panchayat.

Informed consent was obtained from the sample

Findings: Findings of the study were presented under the following headings based on the study objectives

Objective 1 To assess the knowledge, practice and attitude among postnatal mothers in a selected villages adopted by rural health centrepooncheri, Kanchipuram District, Tamil Nadu India.

\section{The finding of the present study reveals that}

$9(12.3 \%)$ of postnatal mothers had adequate knowledge

$38(52 \%)$ of postnatal mothers had moderate knowledge

$26(35.6 \%)$ of postnatal mothers had inadequate knowledge

$1(1.3 \%)$ of postnatal mothers had adequate practice

$28(38.3 \%)$ of postnatal mothers had moderate practice

$44(60.2 \%)$ of postnatal mothers had inadequate practice

$44(60.2 \%)$ of postnatal mothers had negative attitude

$29(39.7 \%)$ of postnatal mothers had undecided attitude

$0(0 \%)$ of postnatal mothers had positive attitude 
Objective 2 To associate demographic variables with the level of knowledge of postnatal exercise.

Finding-1: Age and level of knowledge of postnatal exercise.

There was no significant association between the age and the level of knowledge of postnatal exercise $\mathrm{x}^{2}=$ $12.35, \mathrm{p}(0.05)=12.59$

Finding-2: Education status and level of knowledge of postnatal exercise.

There was no significant association between the Education status and the level of knowledge of postnatal exercise $\mathrm{x}^{2}=8.304, \mathrm{p}(0.05)=18.31$

Finding-3: Occupation and level of knowledge of postnatal exercise

There was no significant association between the occupation and the level of knowledge of postnatal exercise $x^{2}=12.97, p(0.05)=16.81$

Finding-4: Family income and level of knowledge of postnatal exercise

There was no significant association between the family income and the level of knowledge of postnatal exercise $\mathrm{x}^{2}=5.878 \mathrm{p}(0.05)=15.51$

Finding-5: Type of family and level of knowledge of postnatal exercise

There was no significant association between the type of family and the level of knowledgeof postnatal exercise $x^{2}=1.520 p(0.05)=9.49$

Finding-6: Gravida and level of knowledge of postnatal exercise

There was no significant association between the gravida and the level of knowledge of postnatal exercise $\mathrm{x}^{2}=2.734 \mathrm{p}(0.05)=15.51$

Finding-7: Type of delivery and level of knowledge of postnatal exercise

There was no significant association between the type of delivery and the level of knowledge of postnatal exercise $x^{2}=7.819 p(0.05)=9.49$

Finding-8: Live birth and level of knowledge of postnatal exercise
There was no significant association between the live birth and the level of knowledge of postnatal exercise $\mathrm{x}^{2}=4.183 \mathrm{p}(0.05)=15.51$

Finding-9: Source of information and level of knowledge of postnatal exercise

There was no significant association between the source of information and the level of knowledge of postnatal exercise $x^{2}=17.71 p(0.05)=20.09$

To associate demographic variables with the level of Practice of postnatal exercise.

Finding-1: Age and level of Practice of postnatal exercise.

There was no significant association between the age and the level of practice of postnatal exercise $\mathrm{x}^{2}=$ 4.976, $\mathrm{p}(0.05)=12.59$

Finding-2: Education status and level of practice of postnatal exercise .

There was no significant association between the Education status and the level of practice of postnatal exercise $\mathrm{x}^{2}=5.323, \mathrm{p}(0.05)=18.31$

Finding-3: Occupation and level of practice of postnatal exercise

There was no significant association between the occupation and the level of practice of postnatal exercise $\mathrm{x}^{2}=5.370, \mathrm{p}(0.05)=12.59$

Finding-4: Family income and level of practice of postnatal exercise

There was no significant association between the family income and the level of practice of postnatal exercise $\mathrm{x}^{2}=6.852, \mathrm{p}(0.05)=15.51$

Finding-5: Type of family and level of practice of postnatal exercise

There was no significant association between the type of family and the level of practice of postnatal exercise $x^{2}=1.645, p(0.05)=9.49$

Finding-6: Gravida and level of practice of postnatal exercise

There was no significant association between the gravida and the level of practice of postnatal exercise $\mathrm{x}^{2}=2.096, \mathrm{p}(0.05)=15.51$ 
Finding-7: Type of delivery and level of practice of postnatal exercise

There was no significant association between the type of delivery and the level of practice of postnatal exercise $x^{2}=6.852 p(0.05)=9.49$

Finding-8: Live birth and level of practice of postnatal exercise

There was no significant association between the live birth and the level of practice of postnatal exercise $\mathrm{x}^{2}=2.347, \mathrm{p}(0.05)=15.51$

Finding-9: Source of information and level of practice of postnatal exercise

There was no significant association between the source of information and the level of practice of postnatal exercise $\mathrm{x}^{2}=11.96 \mathrm{p}(0.05)=15.51$

To associate demographic variables with the level of Attitude of postnatal exercise.

Finding-1: Age and level of attitude of postnatal exercise.

There was no significant association between the age and the level of attitude of postnatal exercise $x^{2}=$ $6.961, \mathrm{p}(0.05)=12.59$

Finding-2: Education status and level of attitude of postnatal exercise.

There was no significant association between the Education status and the level of attitude of postnatal exercise $\mathrm{x}^{2}=4.739, \mathrm{p}(0.05)=18.31$

Finding-3: Occupation and level of attitude of postnatal exercise

There was no significant association between the occupation and the level of attitude of postnatal exercise $\mathrm{x}^{2}=6.994, \mathrm{p}(0.05)=12.59$

Finding-4: Family income and level of attitude of postnatal exercise

There was no significant association between the family income and the level of attitude of postnatal exercise $x^{2}=13.77 p(0.05)=15.51$

Finding-5: Type of family and level of attitude of postnatal exercise
There was no significant association between the type of family and the level of attitude of postnatal exercise $\mathrm{x}^{2}=0.534 \mathrm{p}(0.05)=9.49$

Finding-6: Gravida and level of attitude of postnatal exercise

There was no significant association between the gravida and the level of attitude of postnatal exercise $\mathrm{x}^{2}=5.093, \mathrm{p}(0.05)=15.51$

Finding-7: Type of delivery and level of attitude of postnatal exercise

There was no significant association between the type of delivery and the level of attitude of postnatal exercise $x^{2}=4.008 p(0.05)=9.49$

Finding-8: Live birth and level of attitude of postnatal exercise

There was no significant association between the live birth and the level of attitude of postnatal exercise $\mathrm{x}^{2}=4.206, \mathrm{p}(0.05)=15.51$

Finding-9: Source of information and level of attitude of postnatal exercise

There was no significant association between the source of information and the level of attitude of postnatal exercise $\mathrm{x}^{2}=3.508 \mathrm{p}(0.05)=15.51$

\section{Conclusion}

To conclude the Researcher would like to: Improve the knowledge, practice and attitude on postnatal exercise among postnatal mothers, create awareness on benefits of postnatal exercise and prevention of complication during postnatal period.

\section{Conflict of Interest: Nill}

Source of Funding: Self funding and no external funding.

Ethical Clearance: Obtained clearance from institutional human ethical committee on 04.02.2018

\section{Reference}

1. Bent V. Ruthilinda. K. Brown; Myles. Text Book for Midwives, 13 edition ed: Churchill Livingstone Publishers; 1999

2. Myles. Textbook for Midwives. Edition fourteenth. 
3. Rajiya. A study on effectiveness of video assisted teaching regarding postnatal exercises among postnatal mother. Banglore; 2011. 4. Shelin Christie. To assess effectiveness of planned teaching programme on knowlegde regarding postnatal exercise among postnatal mothers. Journal of Nursing and Health Sciene Nov-Dec. 2014; 3(6).
5. Swastikmulay. Sinhgad e-journal of nursing dec. 2016; 4(2).

6. Avinash H. Salunkhe. Knowlegde of primigravida mothers regarding postnatal exercise; Indian Journal of Applied Research July 2016; 6(7). 